\title{
Aphyllophoroid fungi (Basidiomycota) in Tunguska River basin, central East Siberia, Russia
}

\author{
HEIKKI KOTIRANTA and ANTON G. SHIRYAEV
}

KOTIRANTA, H. \& SHIRYAEV, A. G. 2015 (2016): Aphyllophoroid fungi (Basidiomycota) in Tunguska River basin, central East Siberia, Russia - Karstenia 55: 25-42. HELSINKI. ISSN 0453-3402.

\begin{abstract}
The forests in Tunguska River basin in central East Siberia were investigated in autumn 2013. The closest study sites of Aphyllophoroid fungi are situated about $650 \mathrm{~km}$ south of Tunguska River in Krasnoyarsk area. Altogether 548 collections and notes were made and 248 taxa identified. The most species rich morph groups were corticioid fungi with 120 taxa, followed by 65 poroid taxa and 63 species of cantharelloid, clavarioid and hericioid fungi. The richest genus was Typhula with 17 species before Hyphodontia sensu lato (10 species), Tubulicrinis (9), Phellinus sensu lato (8) and Phlebia (8). Some short descriptions of untypical or unidentified specimens are given.
\end{abstract}

Key words: central East Siberia, clavarioid fungi, corticioid fungi, poroid fungi

Heikki Kotiranta, Finnish Environment Institute, Biodiversity Unit, P.O. Box 140, Helsinki, FI-00251 Finland; e-mail: heikki.kotiranta@ymparisto.fi

Anton G. Shiryaev, Institute of Plant and Animal Ecology, Vegetation and Mycobiota Biodiversity Department, Ural Division of the Russian Academy of Science, 8 March str. 202,Ekaterinburg, RU-620144 Russia; e-mail: anton.g.shiryaev@gmail.com

\section{Introduction}

The vast area of Siberia and Russian Far East has interested all kinds of biologists. However, the expeditions have mostly concentrated on the Ural Mountains between Asia and Europe in the west (Kotiranta et al. 2005, 2007, Shiryaev et al. 2010), Altay and Sayan Mountains in the south or Russian sea shores (Kotiranta \& Mukhin 2000) plus islands and peninsulas (Parmasto 1963, Kotiranta \& Mukhin 1998). Also the West Siberian and North Siberian plains are rather well investigated (Mukhin 1993, Kotiranta 1995, Mukhin \& Kotiranta 2001, Stavishenko 2011), whereas very little is known of the mycota in central East Siberia and there are no earlier collections of the groups of fungi we studied.
The closest sites where polypores, corticioids, and other Aphyllophoroid fungi have been collected are in the vicinity of the city Krasnoyarsk, about $650 \mathrm{~km}$ south, in Taimyr peninsula about $1300 \mathrm{~km}$ NE, and in Sakha Republic about 2000 $\mathrm{km} \mathrm{E}$ of our study area.

\section{Material and methods}

Altogether 548 Aphyllophoroid specimens were collected or noted during the XXII International Trans-Siberian Mycological Expedition in Tunguska river basin in Au- 
gust 2013. Moreover, amateurs collected here in September 2014 and they gave their specimens to our disposal. Their collections have the number of SVER (see below). Altogether 248 taxa are listed and every species was collected at least once. Species like Fomes fomentarius and Fomitopsis pinicola were mainly noted only (in list, sight).

The study area belongs to the boreal vegetation zone (Ahti et al. 1968), roughly between the northern- and middle boreal subzones. The annual precipitation is 593.1 $\mathrm{mm}$, annual mean temperature $-3.2^{\circ} \mathrm{C}$, mean temperature in January $-23.6{ }^{\circ} \mathrm{C}$ and in July $+18.2{ }^{\circ} \mathrm{C}$, respectively (averages for the period 1981-2010) (Harris et al. 2014).

The study area is in Krasnoyarsk Territory, Evenk Autonomous District, Central Siberian Nature Reserve, Podkammennaja Tunguska River $\left(62^{\circ} 06^{\prime} 52^{\prime \prime} \mathrm{N}, 091^{\circ} 30^{\prime} 03^{\prime \prime}\right.$ E). We also collected in unprotected areas along the river. The forests are partly relatively young, about 60 years old, partly in virgin state. The trees in the area are Alnus sibirica, Betula spp., Larix sibirica, Picea obovata, Pinus sibirica, P. sylvestris, Populus tremula, Prunus padus, Salix caprea, Salix spp. and Sorbus sibirica. The authors of the tree species can be found in Czerepanonov (1995) and are not repeated here. Here we collected one week in thick smoke due to forest- and mire fires (Fig. 1).

The material was identified in Helsinki and Ekaterinburg, and the specimens are deposited in the herbaria of University of Helsinki $(\mathrm{H})$ and/or in the mycological herbarium of the Institute of Plant and Animal Ecology of Ural Division of the Russian Academy of Sciences, Ekaterinburg (SVER) or in the reference herbarium of Heikki Kotiranta (H.K.).

In the text following abbreviations for the substrates are used: $\mathrm{Ab}=$ Abies sibirica, $\mathrm{Al}=$ Alnus sibirica, $\mathrm{B}=$ Betula spp., $\mathrm{D}=$ deciduous wood, $\mathrm{L}=$ Larix sibirica, $\mathrm{Pic}$ $=$ Picea obovata, Psi $=$ Pinus sibirica, Psy $=$ Pinus sylves tris, Pop = Populus tremula, Prunus padus $=$ Pru, Salix caprea $=$ Scap, $\mathrm{S}=$ Salix spp. The collectors are Heikki Kotiranta $(H K)$ and Anton. G. Shiryaev $(A G S)$.

The nomenclature of fungi mostly follows Kotiranta et al. (2009). The genus Hyphodontia J. Erikss. sensu lato is according to Hjortstam \& Ryvarden (2009). Some of the specimens collected do not fit with any species known to us, and then a brief description is given. Also specimens, which deviate somehow from the common species concept, are briefly described. The spores were measured in Cotton Blue (CB), and other mounting media used were $3 \% \mathrm{KOH}$ and Meltzer's reagent (MLZ). $\mathrm{CB}+$ means that this mountant gives a cyanophilous reaction.

The species are arranged into two lists: the first one contains three morphotypes: poroid, corticioid and thelephoroid fungi, and the other one cantharelloid, clavarioid and hericioid fungi. In "Discussion" (below) the commonness or rarity only the species of the List 1 are used, since the abundance of the species in List 2 was not so accurately listed in field. In the lists the taxa are arranged alphabetically regardless of their taxonomic possession.

Old-forest/primeval forest indicators (see Kotiranta \& Niemelä 1996, p. 19-21) are marked (OFI/PFI). We added some species to the list of Kotiranta \& Niemelä (1996), and we believe it reflects better the situation in Russia.

\section{Taxonomy and floristics}

\section{List 1 (polypores, corticioids and Thelephoroid fungi)}

Acanthophysium lividocoeruleum (P. Karst.) Boid. (Aleurodiscus lividocoeruleus (P. Karst.) P.A. Lemke): decorticated Pic, decay 1, diam. 12 cm (HK 26221).

Alutaceodontia alutacea (Fr.) Hjortstam \& Ryvarden (Hyphodontia alutacea (Fr.) J. Erikss.): decorticated Psy, decay 2, diam.13 cm (HK 26113), decorticated Pic, decay 2, diam. $20 \mathrm{~cm}$ (HK 26191), Pic (AGS 58727).

Amphinema byssoides (Pers. : Fr.) J. Erikss.: partly corticated L, decay 4 , diam. $10 \mathrm{~cm}(H K$ 26098), corticated Al, decay 3, diam. $6 \mathrm{~cm}(H K$ 26152), corticated Pic, decay 3 , diam. $9.5 \mathrm{~cm}$ (HK 26163), corticated Pic branch, decay 3, diam. $2.5 \mathrm{~cm}$ (HK 26238), decorticated Pic, decay 1 , diam. $3 \mathrm{~cm}(H K 26245+$ four $\times$ sight $)$, corticated Psi branch, decay 1, diam. $0.7 \mathrm{~cm}(H K$ 26258), Pic (SVER 58419).

Amylocorticium subincarnatum (Peck) Pouzar: decorticated Pic, decay 3, diam. $18 \mathrm{~cm}$ (HK 26173), decorticated Pic, decay 3, diam. $9.5 \mathrm{~cm}$ (HK 26250), decorticated Pic, decay 3, diam. 9.5 $\mathrm{cm}(H K 26250)$. We follow here the species concept of Eriksson \& Ryvarden (1973).

Amyloporia xantha (Fr. : Fr.) Bondartsev (Antrodia xantha (Fr. : Fr.) Ryvarden): moss-covered, decorticated Pic, decay 2, diam. $29 \mathrm{~cm}$ (HK 26076), decorticated Psy branch, decay 2, diam. $8 \mathrm{~cm}$ (HK 26104), (SVER 58648).

Amylostereum chailletii (Pers. : Fr.) Boidin: decorticated L driftwood, decay 1, diam. $21 \mathrm{~cm}$ (HK 26067), Pic (SVER 58507). OFI.

Antrodia serialis (Fr.) Donk: corticated Pic, decay 3, diam. $20 \mathrm{~cm}$ (HK 26143), decorticated Pic, decay 3, diam. $9.5 \mathrm{~cm}$ (HK 26160), decorticated Pic, decay 1, diam. $8 \mathrm{~cm}$ (HK 26179), decorticated Pic, decay 2, diam. $18 \mathrm{~cm}$ (sight), decorticated Pic, decay 3, diam. $16 \mathrm{~cm}$ (HK 26207), decorticated Pic, decay 1, diam. $9.5 \mathrm{~cm}$ (sight), Psy (SVER 58610). 


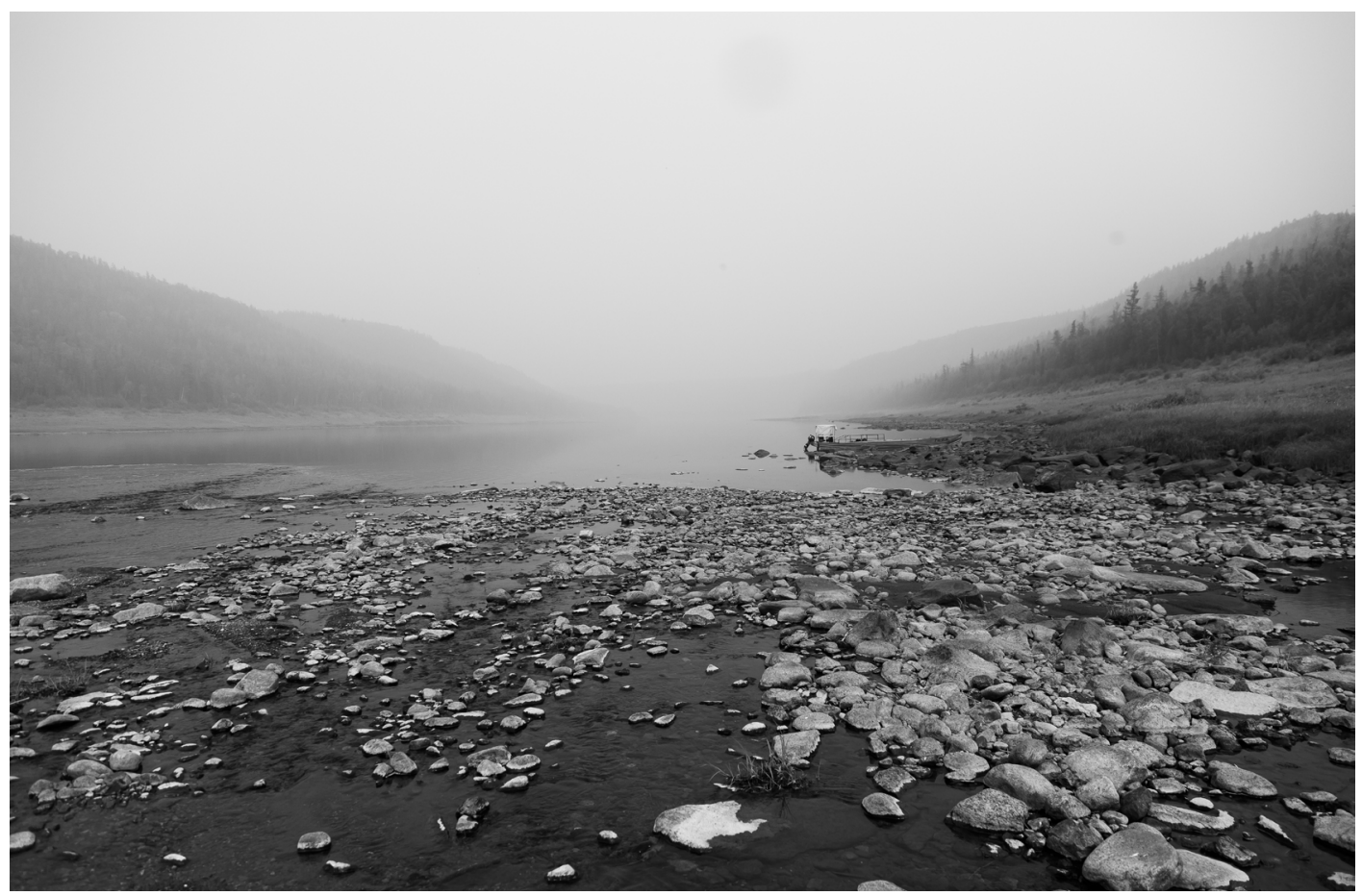

Fig. 1. Podkammennaja Tunguska river ("Stony Tunguska River") in smoke.

Antrodia sinuosa (Fr.) P. Karst.: corticated S (HK 26086). Note: spores allantoid, $6 \times 1.5$ $\mu \mathrm{m}$, decorticated Pic, decay 3 , diam. $18 \mathrm{~cm}(H K$ 26171), decorticated Pic, decay 3, diam. $12 \mathrm{~cm}$ (HK 26220), Psy (SVER 58571).

Antrodiella pallescens (Pilát) Niemelä \& Miettinen ("Antrodiella semisupina" (Berk. \& M.A. Curtis) Ryvarden sensu auct.): corticated B branch, decay 4, diam. $4 \mathrm{~cm}$ (HK 26137).

Athelia bombacina (Pers.) Jül. (HK 26222): decorticated Pic, decay 3, diam. $12 \mathrm{~cm}$. Monomitic, clamped, basal hyphae up to $5 \mu \mathrm{m}$ wide, context hyphae partly encrusted, cystidia none, basidia clavate, often stalked, $(8-) 9.5-13(-15) \times 4-5$ $\mu \mathrm{m}$, with four, up to $6 \mu \mathrm{m}$ long sterigmata, which bend inwards, spores ellipsoid, often in tetrads, $4-5 \times 2-2.9 \mu \mathrm{m}, \mathrm{L}_{\mathrm{m}}=4.4 \mu \mathrm{m}, \mathrm{W}_{\mathrm{m}}=2.5 \mu \mathrm{m}, \mathrm{Q}=$ $1.6-2.1, \mathrm{Q}_{\mathrm{m}}=1.8(\mathrm{n}=20)$.

The spores are smaller, especially narrower, than usually (e.g., Eriksson \& Ryvarden 1973, Bernicchia \& Górjon 2010). The formation of spore tetrads is described in Kotiranta \& Saarenoksa (2005).
Athelia decipiens (Höhn. \& Litsch.) J. Erikss.: decorticated Pic, decay 3, diam. $12 \mathrm{~cm}$ (HK 26222), decorticated Pic, decay 1, diam. $17 \mathrm{~cm}$ (HK 26239).

Athelia epiphylla Pers: : Fr.: Pic (SVER 58522).

Athelopsis sp. (HK 26151): corticated Al, decay 3 , diam. $6 \mathrm{~cm}$. Basidiocarp resupinate, relatively thick, creamish white, easily detachable, cracking when dry, margin porose-reticulate, thinning out; hyphal system monomitic, hyphae clamped, thinwalled, in subiculum parallel to the substrate, 2-3 $\mu \mathrm{m}$ wide, in subhymenium thin-walled, richly clamped, $2 \mu \mathrm{m}$ wide, cystidia none, but a few finger-like hyphidia, 19-25 × 3-4 $\mu \mathrm{m}$, basidia clavate, often internally repetitive, stalked, 16-20 $\times 5-6 \mu \mathrm{m}$, spores pip-shaped to fusoid, with a prominent apiculus, thin-walled, CB-, inamyloid, (4.7-)5-6 × (2.5-)2.8-3 $\mu \mathrm{m}, \mathrm{L}_{\mathrm{m}}=5.3 \mu \mathrm{m}, \mathrm{W}_{\mathrm{m}}=$ $2.9 \mu \mathrm{m}, \mathrm{Q}=1.7-2, \mathrm{Q}_{\mathrm{m}}=1.9(\mathrm{n}=30)$.

The shape of the pip-shaped spores reminds those of Ceraceomyces tessulatus (W.B. Cooke) Jülich, and the fusoid spores those of Leptosporomyces fusoideus (Jülich) Kriegelst. Our speci- 
men differs from the former in having partly differently shaped spores, smaller, often repetitive basidia and narrower hyphae, and from the latter by shorter, but wider spores which are also partly differently shaped, and larger, often repetitive basidia. None of the boreal Athelopsis species known by us (Kotiranta \& Saarenoksa 2005) fits our specimen.

Basidiodendron eyrei (Wakef.) Luck-Allen: decorticated Al, decay 2, diam. $6 \mathrm{~cm}$ (HK 26050), decorticated B branch, decay 1, diam. $4 \mathrm{~cm}(H K$ 26231).

Basidioradulum radula (Fr.) Nobles: corticated Al, decay 2, diam. $2.5 \mathrm{~cm}$ (HK 26119), decorticated Al, decay 3, diam. $6 \mathrm{~cm}$ (HK 26121).

Bjerkandera adusta (Willd. : Fr.) P. Karst.: corticated B (sight), corticated Pop stub, decay 3, diam. $18 \mathrm{~cm}$ (sight), sawed Pop log, decay 2, diam. $18 \mathrm{~cm}$ (HK 26083), dead, erect Pop, decay 2, diam. 13 cm (HK 26147), B, S (SVER 59213).

Boidinia furfuracea (Bres.) Stalpers \& Hjortstam: decorticated Pic, decay 3, diam. $9.5 \mathrm{~cm}(H K$ 26162), decorticated Pic, decay 3, diam. $16 \mathrm{~cm}$ (HK 26209).

Boletopsis grisea (Peck) Bondartsev \& Singer: soil in Psy forest (SVER 59087)

Botryobasidium botryosum (Bres.) J. Erikss. (Botryobasidium vagum (Berk. \& M.A. Curtis) D.P. Rogers sensu auct.): Psy (SVER 58776)

Botryobasidium cf. candicans J. Erikss. (HK 26172c): decorticated Pic, decay 3, diam. $18 \mathrm{~cm}$. Since the specimen has no anamorph, we are not sure if this is $B$. candicans, even if the spores fit with this species.

Botryobasidium intertextum (Schwein.) Jülich \& Stalpers: decorticated Pic, decay 3, diam. $11 \mathrm{~cm}$ (HK 26217).

Botryobasidium subcoronatum (Höhn. \& Litsch.) Donk: decorticated Pic, decay 3, diam. $23 \mathrm{~cm}$ (HK 26073), Pic (SVER 59059).

Byssomerulius albostramineus (Torrend) Hjortstam: decorticated Psy branch, decay 1, diam. 4 cm (HK 26102).
Ceraceomyces microsporus K.H. Larss.: decorticated Psy, decay 2, diam. 7 cm (HK 26100).

Ceraceomyces serpens (Tode : Fr.) Ginns: decorticated Pic, decay 3, diam. $4.5 \mathrm{~cm}$ (HK 26177), partly corticated Pic, decay 1, diam. $12 \mathrm{~cm}(H K$ 26203), B (SVER 58494).

Ceraceomyces tessulatus (Cooke) Jülich: B (SVER 58370).

Ceriporiopsis pseudogilvescens (Pilát) Niemelä \& Kinnunen (HK 26085): corticated S decay 2, diam. $2 \mathrm{~cm}$. Basidiocarp looks more like $C$. resinascens (Romell) Domański, but spores are ellipsoid, $4-4.8 \times 2.4-2.8 \mu \mathrm{m}$.

Cerrena unicolor (Bull. : Fr.) Murrill: dead, erect corticated S, decay 2, diam. $7 \mathrm{~cm}$ (HK 26040), corticated B, decay 2, diam. $9 \mathrm{~cm}$ (HK 26060), corticated B (sight), Al (SVER 58738).

Chondrostereum purpureum (Pers. : Fr.) Pouzar: corticated S, decay 2, diam. $5 \mathrm{~cm}$ (HK 26150), B (AGS 59163).

Coltricia perennis (L. : Fr.) Murrill: soil in Psy dominated forest (SVER 59081).

Coniophora olivacea (Pers. : Fr.) P. Karst.: decorticated Pic, decay 3, diam. $23 \mathrm{~cm}(H K$ 26072), decorticated Pic, decay 1, diam. $21 \mathrm{~cm}$ (HK 26164).

Corticium roseum Pers. : Fr.: corticated Pop branch decay 1, diam. $6 \mathrm{~cm}$ (HK 26080), S (AGS 58977).

Crustoderma corneum (Bourdot \& Galzin) Nakasone: Psy (SVER 59010). PFI

Crustoderma dryinum (Berk. \& M.A. Curtis) Parmasto: corticated Pic, decay 3, diam. $9.5 \mathrm{~cm}$ (HK 26161). OFI

Cylindrobasidium evolvens (Fr. : Fr.) Jülich: corticated S, decay 1, diam. $3 \mathrm{~cm}$ (HK 26262).

Cytidia salicina (Fr.) Burt: corticated S, decay 1, diam. $3 \mathrm{~cm}$ (HK 26261), corticated S twigs, decay 1-2, diam. 1-1.5 cm (sight $\times 3)$, co $\mathrm{S}$ branch (SVER 59135). 
Daedaleopsis confragosa (Bolton : Fr.) Schröt.: dead, erect corticated S, decay 2, diam. $8 \mathrm{~cm}(H K$ 26041), S (SVER 58875).

\section{Daedaleopsis tricolor (Pers.) Bondartsev \&} Singer - Fig. 2

Corticated Al decay 2, diam. $5 \mathrm{~cm}$ (HK 26022), corticated Al decay 2, diam. $2 \mathrm{~cm}$ (HK 26033), corticated B branch, decay 2, diam. $4 \mathrm{~cm}(H K$ 26127), corticated B branch, decay 4, diam. 4 cm (HK 26136), corticated B, decay 2, diam. 5 $\mathrm{cm}$ (sight), corticated B, decay 3, diam. $15 \mathrm{~cm}$ (sight), corticated B (sight), corticated Al, decay 2, diam. $2 \mathrm{~cm}$ (HK 26229), corticated B, decay 3, diam. $4 \mathrm{~cm}$ (HK 26230), B (SVER 58834).

Datronia mollis (Sommerf.) Donk: corticated Al, decay 3, diam. $3 \mathrm{~cm}(H K$ 26030)., corticated B branch, decay 2, diam. $1 \mathrm{~cm}$ (HK 26056), corticated B, decay 3, diam. $5 \mathrm{~cm}$ (HK 26058), corticated $\mathrm{Al}$ (sight), corticated B, decay 1, diam. 3 $\mathrm{cm}$ (sight), corticated S, decay 1, diam. $4 \mathrm{~cm}(H K$ 26227), S, B (SVER 59111).

Datronia scutellata (Schwein.) Gilbn. \& Ryvarden: corticated Al, decay 2, diam. $6.5 \mathrm{~cm}(H K$ 26024), corticated Al, decay 2, diam. $1 \mathrm{~cm}+1.3$ cm (HK 26089), Al (SVER 59225).

Dichomitus squalens (P. Karst.) D.A. Reid: corticated L, decay 2, diam. $30 \mathrm{~cm}$ (HK 26142), L (SVER 59019). OFI.

Dichostereum boreale Pouzar: decorticated, moss-covered Pic, decay 4, diam. $34 \mathrm{~cm}$ (HK 26219).

Fomes fomentarius (L. : Fr.) Fr.: corticated B (HK 26036), dead, erect corticated Pop, decay 3, diam. $11 \mathrm{~cm}$ (HK 26079), corticated, dead erect $\mathrm{B}$, decay 3, diam. $11 \mathrm{~cm}$ (sight), corticated B, decay 2, diam. $9 \mathrm{~cm}$ (sight), B + 45 (sight), B (SVER 58881).

Fomitopsis cajanderi (P. Karst.) Kotl. \& Pouzar: decorticated Pic (dead fruit body, HK 26135c). Pic, L (SVER 58941). OFI.

Fomitopsis pinicola (Sw. : Fr.) P. Karst.: corticated L (HK 26039), dead erect, corticated B, de- cay 3 , diam. $30 \mathrm{~cm}$ (sight), corticated Pic stump +1 (sight), decorticated Pic, decay 1, diam. 26 $\mathrm{cm}$ (sight), corticated Pic, decay 2, diam. $11 \mathrm{~cm}$ (HK 26176), corticated Pic, decay 3, diam. 13 cm (sight), decorticated Pic, decay 4, diam. 16 $\mathrm{cm}($ sight $)$, Pic $\times 6$ (sight), dead erect Psi, decay 2, diam. $45 \mathrm{~cm}$ (sight), decorticated Pic, decay 1, diam. $17 \mathrm{~cm}$ (sight), partly corticated Pic, decay 3, diam. $24 \mathrm{~cm}$ (HK 26242), corticated Pic $\times 3$ (sight), Psy, Pic (SVER 58417).

Fomitopsis rosea (Alb. \& Schwein. : Fr.) P. Karst.: decorticated Pic, decay 3, diam. $23 \mathrm{~cm}$ (dead fruit body, sight), decorticated Pic, decay 3, diam. $20 \mathrm{~cm}$ (HK 26144), decorticated Pic, decay 3, diam. $9.5 \mathrm{~cm}$ (HK 26159), decorticated Pic, decay 3, diam. $18 \mathrm{~cm}$ (dead fruit body, sight), corticated Psi, decay 3, diam. $37 \mathrm{~cm}$ (HK 26184), decorticated Pic, decay 2, diam. $20 \mathrm{~cm}$ (sight), corticated Pic, decay 2, diam. $18 \mathrm{~cm}(H K$ 26204), corticated Pic, decay 3, diam. $16 \mathrm{~cm}$ (HK 26206), corticated Pic, decay 1, diam. $16 \mathrm{~cm}+1$ (sight), decorticated Pic, decay 2, diam. $14 \mathrm{~cm}$ (HK 26225), corticated Pic, decay 1, diam. 14 cm (HK 26246), Pic (SVER 58555). OFI.

Ganoderma applanatum (Pers.) Pat.: corticated B, decay 2, diam. $4.5 \mathrm{~cm}$ ( $H K 26055)$, corticated Al (sight), corticated B, decay 2, diam. $20 \mathrm{~cm}$ (sight), corticated B + 3 (sight), corticated S (sight), B (AGS 58639).

Gloeopeniophorella convolvens (P. Karst.) Boidin, Lanq. \& Gilles: corticated Al, decay 2, diam. $4.5 \mathrm{~cm}(H K 26116)$, decorticated B, decay 3, diam. $12 \mathrm{~cm}$ (HK 26128).

Gloeophyllum protractum (Fr.) Imazeki: Pic (SVER 58564). PFI.

Gloeophyllum sepiarium (Wulfen : Fr.) P. Karst.: L (sight), corticated Psy, decay 2, diam. $12 \mathrm{~cm}$ (HK 26108), Pic (SVER 58659).

Gloeoporus pannocinctus (Romell) J. Erikss. (Ceriporiopsis pannocincta (Romell) Gilbn. \& Ryvarden): corticated B branch, decay 2, diam. $2 \mathrm{~cm}$ (HK 26126). OFI.

Hapalopilus rutilans (Pers. : Fr.) P. Karst. (Hapalopilus nidulans (Fr.) P. Karst.): corticated 


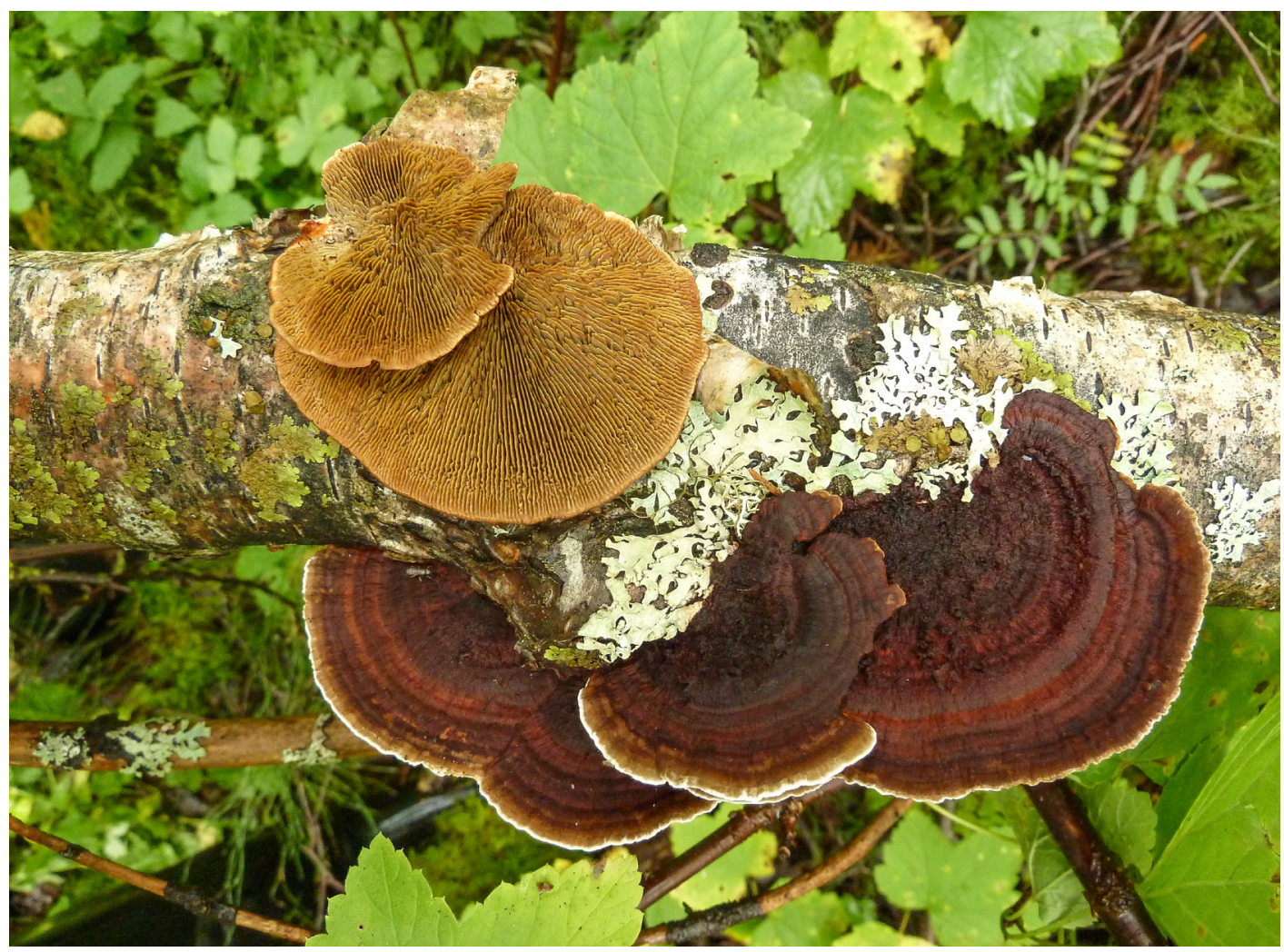

Fig. 2. Conspicuous and beautiful Daedaleopsis tricolor is one of the most common polypores on Betula spp. and Alnus sibirica.

B branch, decay 4, diam. $2 \mathrm{~cm}$ (HK 26129), B (SVER 59017).

Haploporus odorus (Sommerf.) Bondartsev \& Singer: partly living Scap, diam. $18 \mathrm{~cm}$ (HK 26259), dead erect Scap, decay 3, diam. $19 \mathrm{~cm}$ (HK 26260), dead Scap (SVER 58928). OFI.

\section{Hydnomerulius sp.}

- Fig. 3

Decorticated moss-covered Pic, decay 4, diam. $34 \mathrm{~cm}$ (HK 26218): Fruit body resupinate, merulioid when young, later hydnoid - irpicoid with up to $3 \mathrm{~mm}$ long teeth, relatively thick, soft and juicy when fresh, papery and very fragile when dry, loosening easily from the substrate, first bright citric yellow, later bright orange and when mature olive greenish, brown to blackish brown when dry, subiculum pale brownish; hyphal system monomitic, hyphae clamped or simple septate, subicular hyphae clamped, with thickened walls or thin-walled, partly encrusted, sparingly branched, varying much in width, $(2-15 \mu \mathrm{m})$, normally $6-10 \mu \mathrm{m}$ in diam., with conspicuous clamps which often are in pairs, up to $33 \mu \mathrm{m}$ wide, and narrow, $2 \mu \mathrm{m}$ wide hyphae, with low clamps, surround the wider "core" hyphae, tramal hyphae strictly parallel, relatively thin-walled, (2-)7.5-10 $\mu \mathrm{m}$ wide, very sparsely clamped, more often simple septate, sometimes wrinkled, subhymenial hyphae very thin-walled, 2-3 $\mu \mathrm{m}$ wide, cystidia none, but cystidioles scattered, thin-walled, mostly fusiform, apically sometimes prolonged, obtuse, projecting slightly over the basidia, (22-)30-45(-70) $\times$ (6-)8-10 $\mu \mathrm{m}$, basidia clavate to subcylindrical, thin-walled, $(22-) 26-44 \times 4.5-6 \mu \mathrm{m}$, with four thin, up to $5 \mu \mathrm{m}$ long sterigmata, spores ellipsoid to broadly ellipsoid, 3-3.6 × 2-2.9 $\mu \mathrm{m}, \mathrm{L}_{\mathrm{m}}$ 
$=3.2 \mu \mathrm{m}, \mathrm{W}_{\mathrm{m}}=2.5 \mu \mathrm{m}, \mathrm{Q}=1.1-1.6, \mathrm{Q}_{\mathrm{m}}=1.3$ $(n=30)$, with negligible apiculus and thick walls, $\mathrm{CB}+$ (strongly), MLZ pale olive greenish - indistinctly dextrinoid, $\mathrm{KOH}$ hyaline.

Our specimen mostly resembles $H$. pinastri (Fr.) Jarosch \& Besl. (syn. Leucogyrophana pinastri (Fr.) Ginns \& Weresub). Especially the tramal hyphae are very similar in being strictly parallel, sometimes wrinkled, having only a few clamps, and being mostly simple septate. The colour of the fresh fruit body is, however, different. We have never seen $H$. pinastri with such bright yellow and orange colours. Also the spores are small, roughly only a half of those of H. pinastri (Hallenberg 1985). Pseudomerulius montanus (Burt) Kotir., K.H. Larss. \& Kulju, has even smaller spores than our specimen, which are besides dextrinoid, and the cystidia/cystidioles are differently shaped and basidia smaller (Kotiranta et al. 2011).

Hymenochaete "laricis" (HK 26070): living L, diam. $60 \mathrm{~cm}$, at 1.5 meters height in resin flow. The fruit body is basically similar to that of $H$. tabacina, but the cap is smaller and narrower, and setae shorter.

Hymenochaete tabacina (Sowerby) Lév.: corticated Al, decay 1, diam. $2 \mathrm{~cm}(H K 26157)$, corticated Al, decay 2, diam. $6 \mathrm{~cm}$ (HK 26192), S branch (SVER 58908).

Hyphoderma argillaceum (Bres.) Donk: decorticated B, decay 1, diam. $3 \mathrm{~cm}$ (HK 26139).

Hyphoderma mutatum (Peck) Donk : fallen Pop branch (SVER 58790), corticated B branch, decay 2, diam. $1.5 \mathrm{~cm}$ (HK 26232). Hyphae clamped, with thickened walls (in $\mathrm{KOH}$ ), cystidia of two kinds :a) thick-walled (up to $2 \mu \mathrm{m}$ ), apically encrusted metuloids, often with one or two constrictions and an adventitious septa, 50-77 $\times$ 10-15 $\mu \mathrm{m}$, and, b) clavate, sometimes slightly thick-walled gloeocystidia, 50-62 × 8-12.5 $\mu \mathrm{m}$, basidia basally clamped, basally with thickened walls, often with an adventitious septa, clavate, (39-)53-73 × 6-8 $\mu \mathrm{m}$, with four stout, basally up to $3 \mu \mathrm{m}$ wide and up to $12 \mu \mathrm{m}$ long sterigmata, spores cylindrical, sometimes allantoid or gently sigmoid, $(11-) 12-15(-15.5) \times 3.9-5(-5.2) \mu \mathrm{m}$, $\mathrm{L}_{\mathrm{m}}=13 \mu \mathrm{m}, \mathrm{W}_{\mathrm{m}}=4.3 \mu \mathrm{m}, \mathrm{Q}=2.7-4, \mathrm{Q}_{\mathrm{m}}=3(\mathrm{n}=$ 20), with homogenous contents.
The spores are unusually wide and basidia unusually long.

Hyphoderma occidentale (D.P. Rogers) Boidin \& Gilles (Hyphoderma subdefinitum J. Erikss. \& Å. Strid) (HK 26106): decorticated S, decay 4, diam. $3 \mathrm{~cm}$ (HK 26043), decorticated Psy, decay 1, diam. $8 \mathrm{~cm}$. Hyphae clamped, crystals on subhymenium and often on basidial bases, basidia clavate to cylindrical, 31-33 $\times 6-7 \mu \mathrm{m}$, cystidia cylindrical, (53-)70-100 × 6-8 $\mu \mathrm{m}$, projecting up to $60 \mu \mathrm{m}$ above the basidia, some hyphidia between basidia, spores narrowly ellipsoid, some slightly bent, thin-walled, CB-, inamyloid, (8.9-)10.9-13.1(-14) × 4-5 $\mu \mathrm{m}, \mathrm{L}_{\mathrm{m}}=11.9 \mu \mathrm{m}$, $\mathrm{W}_{\mathrm{m}}=4.5 \mu \mathrm{m}, \mathrm{Q}=2.3-3.1, \mathrm{Q}_{\mathrm{m}}=2.7(\mathrm{n}=16, H K$ 26043). The other specimen (HK 26106) has a bit small spores, viz. 9.6-10.5 $(-14.7) \times 3.1-4.5 \mu \mathrm{m}$, $\mathrm{L}_{\mathrm{m}}=10.1 \mu \mathrm{m}, \mathrm{W}_{\mathrm{m}}=3.9 \mu \mathrm{m}, \mathrm{Q}=2.2-3.7, \mathrm{Q}_{\mathrm{m}}=$ $2.6(n=11)$, cystidia cylindrical, $40-74 \times 6-9 \mu \mathrm{m}$.

Hyphoderma setigerum (Fr.) Donk: corticated Al decay 2, diam. $7 \mathrm{~cm}$ (HK 26021), corticated Al decay $3,3 \mathrm{~cm}$ in diam. (HK 26026), corticated Al, decay 2, diam. $4.5 \mathrm{~cm}$ (HK 26117), corticated Al, decay 2, diam. $4 \mathrm{~cm}$ (HK 26120), corticated $\mathrm{Al}$, decay 2, diam. $2.5 \mathrm{~cm}($ sight +1$)$, corticated Psi crown, decay 1, diam. $12 \mathrm{~cm}$ (HK 26254), Al (SVER 58677).

Hyphoderma sibiricum (Parmasto) J. Erikss. \& A. Strid: decorticated Psy branch, decay 3, diam. $4 \mathrm{~cm}$ (HK 26103).

Hyphodontia alutaria (Burt) J. Erikss.: decorticated Pic, decay 3, diam. $18 \mathrm{~cm}$ (HK 26172).

Hyphodontia pallidula (Bres.) J. Erikss.: Pic trunk (SVER 58633).

Hypochnicium bombycinum (Sommerf.) J. Erikss.: sawed Scap, decay 1, diam. $11 \mathrm{~cm}(H K$ 26188).

Inonotus leporinus (Fr.) P. Karst. (Onnia leporina (Fr.) H. Jahn): Pic trunk + 5 (SVER 59115, AGS sight). OFI.

Inonotus obliquus (Pers. : Fr.) Pilát: living B, diam. $20 \mathrm{~cm}$ (sterile conk, sight), dead, erect B, decay 2, diam. $26 \mathrm{~cm}$ (fertile fruit body, $H K$ 26063), B (SVER 58736). 
Inonotus radiatus (Sowerby : Fr.) P. Karst.: corticated Al, decay 2, diam. $6.5 \mathrm{~cm}$ (HK 26025), corticated Al decay 2, diam. $12 \mathrm{~cm}$ (HK 26028), corticated Al, decay 2, diam. $8 \mathrm{~cm}$ (HK 26047), corticated Al, decay 2, diam. $4 \mathrm{~cm}$ (sight), corticated Al, decay 3, diam. $3.5 \mathrm{~cm}$ (sight), $\mathrm{Al}+3$ (sight), Al (SVER 58946).

Inonotus tomentosus (Fr.) Teng (Onnia tomentosa (Fr.) P. Karst.): ground ( $H K$ 26087), ground, base of fallen Pic (SVER 58992).

Irpex lacteus (Fr. : Fr.) Fr.: corticated B branch, decay 2, diam. $3 \mathrm{~cm}$ (HK 26134), B, Pru (SVER 59094).

Ischnoderma benzoinum (Wahlenb. : Fr.) P. Karst.: decorticated Pic, decay 3, diam. $23 \mathrm{~cm}$ (HK 26071).

Kavinia alboviridis (Morgan) Gilb. \& Budington: base of Pic, and on roots (SVER 58501).

Kneiffiella barba-jovis (Bull. : Fr.) P. Karst. (Hyphodontia barba-jovis (Bull. : Fr.) J. Erikss.): corticated Al, decay 3, diam. $3.5 \mathrm{~cm}$ (HK 26154), Pop trunk (SVER 58756).

Kneiffiella subalutacea (P. Karst.) Jülich \& Stalpers (Hyphodontia subalutacea (P. Karst.) J. Erikss.): Pic branch (SVER 58604).

Laetiporus sulphureus (Bull. : Fr.) Murrill: decorticated L (HK 26193), dead erect L (SVER 58922).

Laricifomes officinalis (Vill. : Fr.) Kotl. \& Pouzar (Fomitopsis officinalis (Vill. : Fr.) Bondartsev \& Singer): L (SVER 59066), high up on living large L (AGS sight). PFI.

Laurilia sulcata (Burt) Pouzar: moss-covered, decorticated Pic, decay 2, diam. $23 \mathrm{~cm}(H K$ 26078), corticated L, decay 3, diam. $18 \mathrm{~cm}(H K$ 26096), corticated L, decay $1+2$, diam. $20 \mathrm{~cm}$ (HK 26097), Pic (SVER 59042). PFI.

Leptoporus mollis (Pers.) Quél.: Pic, diam. 30 cm (SVER 59109). OFI.

Leptosporomyces galzinii (Bourdot) Jülich: decorticated Psy, decay 1, diam. 7 cm (HK 26099), decorticated Psy, decay 2, diam. $13 \mathrm{~cm}$ (HK 26114).

Leptosporomyces mutabilis (Bres.) Krieglst.: decorticated Psi, decay 3, diam. $34 \mathrm{~cm} \mathrm{(HK}$ 26256). Hyphae clamped, especially subhymenial hyphae heavily encrusted, basal ones up to $5 \mu \mathrm{m}$ wide, relatively thin-walled, $\mathrm{CB}+$, cystidia none, but some hyphidia between basidia, 15-20 $\times 3$, basidia subcylindrical to clavate, (7.5-)9-14 $\times(3.5-) 4 \mu \mathrm{m}$, spores ellipsoid to short cylindrical, 3-4.1 × 1.9-2.4 $\mu \mathrm{m}, \mathrm{L}_{\mathrm{m}}=3.5 \mu \mathrm{m}, \mathrm{W}_{\mathrm{m}}=2.1$ $\mu \mathrm{m}, \mathrm{Q}=1.4-2.1, \mathrm{Q}_{\mathrm{m}}=1.7(\mathrm{n}=30)$, thin-walled, faintly $\mathrm{CB}+$.

Leptosporomyces sp. (HK 26172a): decorticated Pic, decay 3 , diam. $18 \mathrm{~cm}$. Basidiocarp white, hypochnoid, loosely attached to the substrate, porose-reticulate under the lens $(\times 50)$, margin not differentiated, distinct; hyphal system monomitic, all septa with clamps, in subiculum thin strands composed of 4-6 hyphae, contextual hyphae without any particular orientation, without crystals, thin-walled, $\mathrm{CB}+, 2-2.5(-3) \mu \mathrm{m}$ wide, cystidia none, basidia clavate, stalked, 9-11 $\times$ $3.5-4 \mu \mathrm{m}$, with four, up to $2 \mu \mathrm{m}$ long sterigmata, spores ellipsoid to broadly ellipsoid, thin- to slightly thick-walled, $\mathrm{CB}+$ (faintly), 2.5-3.1($3.3) \times 2-2.5 \mu \mathrm{m}, \mathrm{L}_{\mathrm{m}}=2.9 \mu \mathrm{m}, \mathrm{W}_{\mathrm{m}}=2.2 \mu \mathrm{m}, \mathrm{Q}$ $=1.1-1.5 \mu \mathrm{m}, \mathrm{Q}_{\mathrm{m}}=1.3(\mathrm{n}=30)$.

The shape of the spores is very similar to that of Trechispora confinis (Bourdot \& Galzin) Liberta, but smaller in our specimen. Moreover the basidia of $T$. confinis are differently shaped, hyphae wider with ampullaceous septa and with crystals. Species of the genus Tretomyces K.H. Larss., Kotir. \& Saaren. have basically spores of the same size and shape, but the hyphae are partly simple septate, partly clamped (Kotiranta et al. 2011) unlike our specimen.

Megalocystidium cf. leucoxanthum (Bres.) Jülich: corticated $\mathrm{Al}$ branch, decay 2, diam. $2 \mathrm{~cm}$ (HK 26023), corticated Al decay 1, diam. 5.5 $\mathrm{cm}$ (HK 26195). Fruit body resupinate, smell of anise when fresh (like in M. leucoxanthum), hyphae clamped, gloeocystidia long, spores ellipsoid, sometimes adaxial side concave, thinwalled, amyloid, (11.3-)12-18.5(-19) × (6.2)7.6-11(-11.6) $\mu \mathrm{m}, \mathrm{L}_{\mathrm{m}}=16.1 \mu \mathrm{m}, \mathrm{W}_{\mathrm{m}}=9.1 \mu \mathrm{m}$, $\mathrm{Q}=1.3-2.1, \mathrm{Q}_{\mathrm{m}}=1.8(\mathrm{n}=30)$.

The spore width and shape does not fit well 


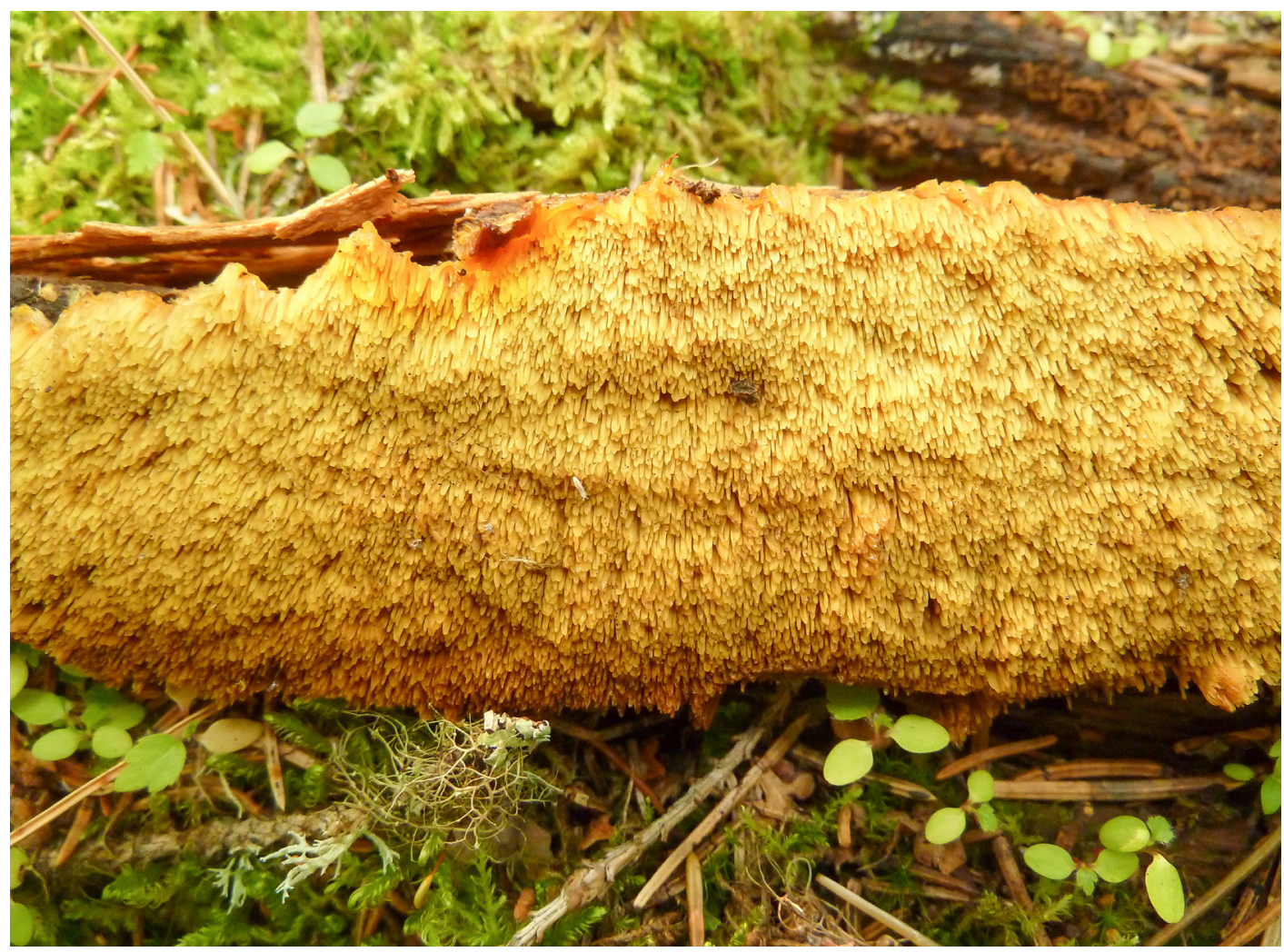

Fig. 3. Hydnomerulius sp. on thick Picea obovata.

with M. leucoxanthum f. salicis (see Eriksson \& Ryvarden 1975, p. 427), but better with Gloeocystidiellum leucoxanthum (Bres.) Boidin var. brevisporum Parmasto, even if in the latter the spores are smaller, viz. $12-14(-14.5) \times 6-7 \mu \mathrm{m}$ (Parmasto 1965).

Mycoacia fuscoatra (Fr.) Nakasone (Phlebia fuscoatra (Fr. : Fr.) Nakasone): Al (SVER 58886), corticated Al, decay 2, diam. $2.5 \mathrm{~cm}$. Spores a bit small, viz. 4.5-5.2 × 1.8-1.9 $\mu \mathrm{m}$, and needle-like cystidioles few (HK 26051), decorticated B, decay 4, diam. $18 \mathrm{~cm}(H K 26062 a)$, spores small, like in $H K 26051$.

Oligoporus sericeomollis (Romell) Bondartsev (Postia sericeomollis (Romell) Jülich): mosscovered, decorticated Pic, decay 2, diam. $29 \mathrm{~cm}$ (HK 26074), decorticated Psy, decay 2, diam. 8 cm (HK 26105), decorticated Psy, decay 3, diam. $6 \mathrm{~cm}$ (HK 26110), Pic trunk (SVER 58696). OFI.
Osteina obducta (Berk.) Donk (Oligoporus obductus (Berk.) Gilbn. \& Ryvarden): L trunk (HK 26199, (SVER 58892). Fruit body like described by Ryvarden and Gilbertson (1994) and Ryvarden and Melo (2014) but our specimen deviates in pore- and spore size from those descriptions. Our specimen has very small pores, viz. 8-11/mm (Ryvarden \& Gilbertson, and Ryvarden $\&$ Melo, $3-5 / \mathrm{mm}$ ) and narrow, up to $2 \mu \mathrm{m}$ wide spores (Ryvarden \& Gilbertson, and Ryvarden \& Melo, 2-2.5 $\mu \mathrm{m})$. Hyphal system monomitic, all hyphae clamped, context hyphae refractive, with thickened walls, $6-10(-12) \mu \mathrm{m}$ in diam., tramal hyphae thin-walled, parallel, 2-3 $\mu \mathrm{m}$ wide, dissepimental hyphae smooth, $1.5 \mu \mathrm{m}$ wide, cystidia none, basidia subcylindrical to clavate, basally clamped, $12-15(-17) \times 4-5 \mu \mathrm{m}$, with four thin, up to $4 \mu \mathrm{m}$ long sterigmata, spores cylindrical to subfusiform (the longest ones), often slightly bent, 4-5.2(-6) $\times 1.5-2 \mu \mathrm{m}, \mathrm{L}_{\mathrm{m}}=4.8 \mu \mathrm{m}, \mathrm{W}_{\mathrm{m}}=$ $1.8 \mu \mathrm{m}, \mathrm{Q}=2.3-3.3, \mathrm{Q}_{\mathrm{m}}=2.7(\mathrm{n}=30)$. OFI. 
Peniophora aurantiaca (Bres.) Höhn. \& Litsch.): corticated Al, decay 1, diam. $2 \mathrm{~cm}$ (HK 26019), corticated Al branch, decay 1, diam. $1.5 \mathrm{~cm}(H K$ 26027), corticated Al branch, decay 1, diam. 1.2 $\mathrm{cm}$ (HK 26031), corticated Al, decay 1, diam. 1.5 cm (HK 26032), corticated Al branch, decay 2, diam. $2 \mathrm{~cm}(H K 26052), \mathrm{Al} \times 18$ (sight), corticated Al branch, decay 2, diam. $0.8 \mathrm{~cm}$ (HK 26190), corticated Al branches, decay 1-2, diam. 0.5-1.2 cm (HK 26228), Al branch (SVER 58721).

Peniophora incarnata (Pers. : Fr.) P. Karst.: corticated B, decay 1, diam. $3 \mathrm{~cm}$ (HK 26141).

Peniophora laurentii S. Lundell: Pop branches (SVER 58687).

Peniophora nuda (Fr.) Bres.: corticated Pop branch, decay 1, diam. $1 \mathrm{~cm}(H K 26081)$.

Peniophora pithya (Pers.) J. Erikss.: corticated L branch, decay 1, diam. $2.5 \mathrm{~cm}$ (HK 26094), Pic branch (SVER 59034).

Peniophora violaceolivida (Sommerf.) Massee (HK 26196): corticated B branch, decay 2, diam. $0.8 \mathrm{~cm}$. Betula is a rare substrate for $P$. violaceolivida, but there are specimens both from Europe and North America, from this substrate (Yurchenko 2010).

Peniophorella pallida (Bres.) K.H. Larss. (Hyphoderma pallidum (Bres.) Donk): decorticated Pic, decay 1, diam. $8 \mathrm{~cm}$ (HK 26251), Al branch (SVER 58983).

Peniophorella praetermissa (P. Karst.) K.H. Larss. (Hyphoderma praetermissum (P. Karst.) J. Erikss. \& Å. Strid): decorticated L driftwood, decay 1, diam. 21 (HK 26068), decorticated Pic, on pore surface of dead F. cajanderi ( $H K$ 26145a), decorticated Pic, decay 2, diam. $18 \mathrm{~cm}$ (HK 26173), decorticated Pic, decay 3, diam. 5 cm (HK 26178), corticated Psi, decay 3, diam. 37 $\mathrm{cm}$ (HK 26185), decorticated Pic, decay 3, diam. $16 \mathrm{~cm}$ (HK 26210).

Phaeolus schweinitzii (Fr.) Pat.: Pic roots (HK 26235). OFI.
Phanerochaete sanguinea (Fr.) Pouzar: corticated L twigs, decay 2, diam. $0.4 \mathrm{~cm}+0.8 \mathrm{~cm}$ (HK 26091).

Phanerochaete tuberculata (P. Karst.) Parmasto: corticated Al, decay 3, diam. $3.5 \mathrm{~cm}$ (HK 26049).

Phanerochaete velutina (DC. : Fr.) P. Karst.: D (HK 26223), B branch (SVER 58841).

Phellinus abietis (P. Karst.) Jahn: living corticated Pic, diam. $9 \mathrm{~cm}$ (HK 26201), Pic trunk (SVER 58764), L (SVER 58743). OFI.

Phellinus alni (Bondartsev) Parmasto: corticated, dead, erect Al (sight), decorticated Al, decay 3 , diam. $3.5 \mathrm{~cm}$ (sight), corticated Al, decay 2, diam. $4.5 \mathrm{~cm}$ (HK 26190).

Phellinus cinereus (Niemelä) M. Fischer: living B, diam. $25 \mathrm{~cm}+1$ (sight)

Phellinus ferrugineofuscus (P. Karst.) Bourdot: corticated L, decay 2, diam. $30 \mathrm{~cm}$ (HK 26090), corticated Pic, decay 3, diam. $16 \mathrm{~cm}$ (sight), corticated L ( $H K$ 26234), corticated Pic, decay 1, diam. $14 \mathrm{~cm}$ (HK 26247), L trunk, diam. $35 \mathrm{~cm}$ (SVER 59001). OFI.

Phellinus lundellii Niemelä: corticated Al, decay 2, diam. $3 \mathrm{~cm}$ (HK 26153). OFI.

Phellinus pini (Brot. : Fr.) A. Ames.: Psy (SVER 58677), living Psi, diam. $40 \mathrm{~cm}$ (HK 26186). Pores $2-4 / \mathrm{mm}$, spores broadly ellipsoid, $4.2-5.2$ $\times 3.6-4 \mu \mathrm{m}$, setae relatively sharp-pointed, 50 $70 \times 9-10 \mu \mathrm{m}$. OFI.

Phellinus punctatus (P. Karst.) Pilát: dead, erect corticated S, decay 2, diam. $20 \mathrm{~cm}$ (HK 26042), corticated Al, decay 2, diam. $8 \mathrm{~cm}$ (HK 26046), corticated Al, decay 1, diam. $6 \mathrm{~cm}$ (HK 26118), S (SVER 59105).

Phellinus tremulae (Bondartsev) Bondartsev \& P.N. Borisov: living Pop, diam. $18 \mathrm{~cm}$ (HK 26082), dead, erect Pop, decay 2, diam. $13 \mathrm{~cm}$ (HK 26146), living Pop (SVER 58995).

Phlebia centrifuga P. Karst.: Pic trunk (SVER 58570). PFI. 
Phlebia gigantea (Fr. : Fr.) Donk: decorticated Pic, decay 1, diam. $26 \mathrm{~cm}$ (HK 26077).

Phlebia cf. livida (Pers. : Fr.) Bres. (HK 26211): decorticated Pic, decay 3 , diam. $16 \mathrm{~cm}$. The fruit body is like "normal" P. livida, but we could not find any cystidia and the spores are small, viz. $(3-) 3.2-4(-4.2) \times 1.6-2.1 \mu \mathrm{m}, \mathrm{L}_{\mathrm{m}}=3.7 \mu \mathrm{m}, \mathrm{W}_{\mathrm{m}}$ $=1.8 \mu \mathrm{m}, \mathrm{Q}=1.7-2.5, \mathrm{Q}_{\mathrm{m}}=2(\mathrm{n}=30)$.

The shape of the spores is somewhere between P. lilascens (Bourd.) Erikss. \& Hjortst. and P. livida, even if most of them are a bit curved. Phlebia tuberculata (Hallenb. \& E. Larss.) GhobadNejhad is microscopically similar to P. livida and grows almost exclusively on hardwoods (Ghobad-Nejhad \& Hallenberg 2010) and its colour does not fit our specimen.

Phlebia nitidula (P. Karst.) Ryvarden: S branch (SVER 58809).

Phlebia radiata Fr.: B branch (SVER 58913).

Phlebia segregata (Bourdot \& Galzin) Parmasto: conifer (SVER 59098).

Phlebia subulata J. Erikss. \& Hjortstam: decorticated Pic, decay 3, diam. $11 \mathrm{~cm}$ ( HK 26216). OFI.

Phlebia tremellosa (Schrad. : Fr.) Nakasone: corticated B, decay 3, diam. $3 \mathrm{~cm}$ (HK 26133), B (SVER 58691).

Phlebiella sulphurea (Pers. : Fr.) Ginns \& M.N.L. Lefebre: corticated L branch, decay 1, diam. $4 \mathrm{~cm}$ (HK 26092), corticated Psy branch, decay 1, diam. $2 \mathrm{~cm}$ (sight), decorticated Pic $\times 3$ (sight), Psy (SVER 58847).

Piptoporus betulinus (Bull. : Fr.) P. Karst.: dead, corticated B (HK 26035), corticated, dead erect B, decay 3, diam. $11 \mathrm{~cm}$ (sight), B (SVER 58795).

Plicatura nivea (Sommerf.) P. Karst.: Al branch (SVER 59234).

Polyporus brumalis (Pers.) Fr.: B branch, Pru branch (SVER 59052).

Polyporus leptocephalus (Jacq. : Fr.) Fr. (Polyporus varius $\mathrm{Fr}$.): corticated $\mathrm{Al}$, decay 2, diam.
$2 \mathrm{~cm}$ (HK 26034), decorticated B twig ( $H K$ 26054), corticated B branch, decay 3, diam. 0.6 $\mathrm{cm}$ (HK 26130), corticated B branch, decay 3, diam. $1 \mathrm{~cm}(H K 26158)$.

Polyporus pseudobetulinus (Pilát) Thorn, Kotir. \& Niemelä: corticated living Pop, diam. $40 \mathrm{~cm}$ (HK 26264, SVER 58859). PFI.

Postia leucomallella (Murrill) Jülich: decorticated Psy, decay 2, diam. $10 \mathrm{~cm}$ ( $H K$ 26111), decorticated Pic, decay 3, diam. $18 \mathrm{~cm}(H K$ 26170), decorticated Pic, decay 4, diam. $16 \mathrm{~cm}$ (HK 26198), decorticated Pic, decay 1, diam. 12 cm (HK 26202), decorticated Pic, decay 2, diam. $10 \mathrm{~cm}$ (HK 26236). OFI.

Repetobasidium erikssonii Oberw. (HK 26069): decorticated L driftwood, decay 1, diam. $21 \mathrm{~cm}$. Basidiocarp very thin, whitish, porose reticulate under the lens $(\times 50)$. Hyphal system monomitic, hyphae thin-walled, clamped, 2-3(-4) $\mu \mathrm{m}$ in diam. Cystidia cylindrical to tubular, multicelled with small clamps, apical cell (15-)20 $31(-36) \times 4-5 \mu \mathrm{m}$, basidia repetitive, basally clamped, pyriform or subglobose, $5-6 \times 4-5 \mu \mathrm{m}$, spores ellipsoid to pip-shaped sometimes adaxially slightly concave, often glued in pairs-tetrads, thin-walled, CB-, inamyloid, (3-)3.3-4.6 $\times$ $2-2.6(-2.8) \mu \mathrm{m}, \mathrm{L}_{\mathrm{m}}=3.9 \mu \mathrm{m}, \mathrm{W}_{\mathrm{m}}=2.3 \mu \mathrm{m}, \mathrm{Q}=$ $1.2-2.2, \mathrm{Q}_{\mathrm{m}}=1.7(\mathrm{n}=20)$.

Our specimen has smaller basidia and spores than reported by Eriksson et al. (1981).

Pseudomerulius aureus (Fr.) Jülich: Psy, diam. $35 \mathrm{~cm}$ (SVER 58618).

Pseudotomentella nigra (Höhn. \& Litsch.) Svrček: B trunk, diam. 30 cm (SVER 59005).

Resinicium furfuraceum (Bres.) Parmasto: partly corticated Pic, decay 3, diam. $13 \mathrm{~cm}$ (HK 26180), decorticated Pic, inside brown-rotted trunk, decay 4, diam. $35 \mathrm{~cm}$ (HK 26200).

Rhodonia placenta (Fr.) Niemelä, K.H. Larss. \& Schigel (Oligoporus placentus (Fr.) Gilbn. \& Ryvarden): decorticated Pic, decay 2, diam. 18 cm (HK 26205). OFI.

Rigidoporus crocatus (Pat.) Ryvarden (HK $26062 b$ ): decorticated B, decay 4, diam. $18 \mathrm{~cm}$. 
Fruit body in poor condition, and only one spore seen, $5 \times 4.6 \mu \mathrm{m}$. OFI.

Scopuloides rimosa (W.B. Cooke) Jülich: decorticated D, decay 3, diam. $4 \mathrm{~cm}$ (HK 26088), decorticated Al, decay 4, diam. $3 \mathrm{~cm}$ (HK 26124), corticated B, decay 2, diam. $2 \mathrm{~cm}$ (HK 26131).

Scytinostroma galactinum (Fr.) Donk: decorticated B branch (SVER 58797).

Sistotrema brinkmannii (Bres.) J. Erikss.: corticated B, decay 1, diam. $3 \mathrm{~cm}$ (HK 26140), corticated B branch (SVER 58676).

Sistotrema diademiferum (Bourdot \& Galzin) Donk: decorticated Pop, decay 4, diam. $7 \mathrm{~cm}$ (HK 26149).

Sistotrema oblongisporum M.P. Christ. \& Hauerslev: corticated Al branch, decay 2, diam. $1 \mathrm{~cm}$ (HK 26125), corticated S, decay 1, diam. $3 \mathrm{~cm}$ (HK 26263).

Sistotrema octosporum (J. Schröt. ex Höhn. \& Litsch.) Hallenb.: decorticated Psy branch, decay 1, diam. $2.5 \mathrm{~cm}$ (HK 26115).

Sistotremastrum niveocremeum (Höhn. \& Litsch.) J. Erikss.: Pic stump (SVER 59072).

Sistotremastrum suecicum Litsch. ex J. Erikss.: Psy trunk (SVER 59122). OFI.

Sistotremella aff. perpusilla Hjortstam (HK 26108): decorticated Psy, decay 3, diam. 9 cm. Basidiocarp very thin, almost invisible, pruinose. Hyphae with low clamps, 1.3-2 $\mu \mathrm{m}$ in diam., cystidia none, basidia urniform, $8-10 \times 4-5 \mu \mathrm{m}$, with 6-8 sterigmata, spores broadly ellipsoid to short ellipsoid, often glued together, thick-walled, $\mathrm{CB}+, 2.5-3.6 \times 1.8-2.3(-2.6) \mu \mathrm{m}, \mathrm{L}_{\mathrm{m}}=2.9 \mu \mathrm{m}$, $\mathrm{W}_{\mathrm{m}}=2 \mu \mathrm{m}, \mathrm{Q}=1.2-1.6, \mathrm{Q}_{\mathrm{m}}=1.5(\mathrm{n}=30)$.

The spores are slightly smaller than reported by Eriksson et al. (1984) or Kotiranta \& Saarenoksa (1990) and therefore we believe, that this might be a taxon of its own, but close to $S$. perpusilla.

Skeletocutis biguttulata (Romell) Niemelä: decorticated Psy, decay 3, diam. $10 \mathrm{~cm}$ (HK 26112).
Skeletocutis kuehneri A. David: decorticated Pic, decay 3, diam. $16 \mathrm{~cm}$ (HK 26208).

Skeletocutis odora (Sacc.) Ginns: decorticated Psi, decay 3, diam. $34 \mathrm{~cm}$ (HK 26255). OFI.

Steccherinum fimbriatum (Pers. : Fr.) J. Erikss.: corticated Al, decay, diam. $3 \mathrm{~cm}$ (HK 26123), corticated Al, decay 3, diam. $4 \mathrm{~cm}$ (HK 26224), B (SVER 58933).

Steccherinum ochraceum (Pers.) Gray coll.: decorticated Al, decay 4, diam. $8 \mathrm{~cm}$ (HK 26122), corticated B, decay 3, diam. $15 \mathrm{~cm}$ (HK 26138), S (SVER 58748).

Steccherinum tenuispinum Spirin, Zmitr. \& Malysheva: decorticated Pic, decay 3, diam. 13 $\mathrm{cm}$ (HK 26183). The fruit body was growing close to dead Fomitopsis pinicola.

Stereum hirsutum (Willd. : Fr.) Gray: decorticated deciduous driftwood, decay 2, diam. $20 \mathrm{~cm}$ (HK 26064), B trunk (SVER 58684).

Stereum sanguinolentum (Alb. \& Schwein. : Fr.) Fr.: corticated Psi crown, decay 1, diam. $12 \mathrm{~cm}$ (HK 26252), Psy (SVER 59176).

Subulicystidium longisporum (Pat.) Parmasto: decorticated B branch, decay 1, diam. $3 \mathrm{~cm}(H K$ 26053), decorticated $\mathrm{B}$, decay 4 , diam. $6.5 \mathrm{~cm}$ (HK 26061), inside brown-rotted, decorticated Pic, decay 4, diam. $19 \mathrm{~cm}$ (HK 26197).

Thelephora palmata (Scop.) Fr.: soil in mixed forest (SVER 58990).

Thelephora terrestris Ehrh. : Fr.: soil in Psy dominated forest (SVER 59048).

Tomentella atramentaria Rostr.: B trunk (SVER 59063).

Tomentella bryophila (Pers.) M.J. Larsen: Pop trunk (SVER 58655).

Tomentella cinerascens (P. Karst.) Höhn. \& Litsch.: Al branch (SVER 58741).

Tomentella crinalis (Fr.) M.J. Larsen: B trunk (SVER 58813). 
Tomentella ellisii (Sacc.) Jülich \& Stalpers: B branches and soil (SVER 58582).

Tomentella ferruginea (Pers.) Pat.: litter with $\mathrm{L}$ and $\mathrm{B}$ branches, leaves and grasses (SVER 58680).

Tomentella radiosa (P. Karst.) Rick: B, Psy branches (SVER 59132).

Tomentella sublilacina (Ellis \& Holw.) Wakef.: Pop branches and litter (SVER 59025).

Tomentellopsis echinospora (Ellis) Hjortstam: Psy and mosses (SVER 58836).

Trametes hirsuta (Wulfen : Fr.) Pilát: corticated Al, decay 3, diam. $4 \mathrm{~cm}$ (HK 26029b), Pru, S (SVER 59191).

Trametes ochracea (Pers.) Gilbn. \& Ryvarden: cut Pop log, decay 2, diam. $18 \mathrm{~cm}$ (HK 26084), sawed Scap, decay 1, diam. $11 \mathrm{~cm}$ (HK 26187).

Trametes pubescens (Schumach. : Fr.) Pilát: corticated B branch, decay 2, diam. $1 \mathrm{~cm}(H K$ 26057), B, S, (SVER 58918).

Trametes suaveolens (Fr.) Fr.: corticated S (SVER 58671).

Trametes velutina (Fr.) G. Cunningh.: corticated B branch, decay 2, diam. $1 \mathrm{~cm}$ (HK 26233).

Trametes versicolor (L. : Fr.) Pilát: corticated Al, decay 2, diam. $4.5 \mathrm{~cm}$ (HK 26045), B, Pop (SVER 58567).

Trechispora farinacea (Pers. : Fr.) Liberta: corticated Psy, decay 2, diam. $7 \mathrm{~cm}$ (HK 26101).

Trichaptum abietinum (Pers. : Fr.) Ryvarden: Pic (sight), corticated L branch, decay 3 , diam. $3 \mathrm{~cm}$ (HK 26038), corticated L, decay 1, diam. $11 \mathrm{~cm}$ (HK 26095), corticated Pic, decay 2, diam. 11 cm (HK 26175), corticated Pic, decay 3, diam. $13 \mathrm{~cm}$ (HK 26181), corticated Pic branch, decay 2, diam. $4 \mathrm{~cm}$ (sight), corticated Psi crown, decay 1, diam. $12 \mathrm{~cm}$ (HK 26253), corticated Psi branch, decay 2, diam. $0.5 \mathrm{~cm}$ ( $H K$ 26257), L (SVER 58701).
Trichaptum fuscoviolaceum (J.C. Schmidt : Fr.) Kreisel: decorticated L driftwood, decay 1, diam. $21 \mathrm{~cm}$ (HK 26065), corticated Psy, decay 2, diam. $12 \mathrm{~cm}$ (HK 26107), Psy (SVER 59202).

Trichaptum laricinum (P. Karst.) Ryvarden: corticated L (HK 26037), L (SVER 58371).

Trichaptum pargamenum (Fr.) G. Cunn.: B branch (SVER 58430).

Tubulicrinis angustus (D.P. Rogers \& Weresub) Donk: Psy trunk, diam. $40 \mathrm{~cm}$ (SVER 58773).

Tubulicrinis borealis J. Erikss.: conifer (SVER 58954).

Tubulicrinis calothrix (Pat.) Donk: decorticated Pic (HK 26145b), decorticated Pic, decay 2, diam. $14 \mathrm{~cm}$ (HK 26226), partly corticated Pic branch, decay 2, diam. 4 cm (HK 26237).

Tubulicrinis confusus K.H. Larss. \& Hjortstam: Psy trunk (SVER 59013).

Tubulicrinis glebulosus (Bres.) Donk: decorticated B, decay 3 , diam. $3 \mathrm{~cm}$. Cystidia 70-100 $\times$ 9-10 $\mu \mathrm{m}$, spores allantoid, 6.4-6.7 × 1.6-1.8 $\mu \mathrm{m}$ (HK 26059), decorticated B, decay 2, diam. $2 \mathrm{~cm}$ (HK 26132), decorticated Pop, decay 1, diam. 7 cm (HK 26148).

Tubulicrinis medius (Bourdot \& Galzin) Oberw.: Psy trunk (SVER 59126).

Tubulicrinis propinguus (Bourdot \& Galzin) Donk: Psy trunk (SVER 58898).

Tubulicrinis sororius (Bourdot \& Galzin) Oberw.: Psy trunk (SVER 58636).

Tubulicrinis subulatus (Bourdot \& Galzin) Donk: corticated Pic, decay 1, diam. $21 \mathrm{~cm}(H K$ 26165), decorticated Pic, decay 2, diam. $3 \mathrm{~cm}$ (HK 26248), L (SVER 58513).

Tulasnella violea (Quél.) Bourdot \& Galzin (HK 26048): corticated $\mathrm{Al}$, decay 3 , diam. $3.5 \mathrm{~cm}$. Simple septate, basidia $8-11 \mu \mathrm{m}$ in diam., sp broadly ellipsoid-subglobose, $8-8.5 \times 6 \mu \mathrm{m}$. 
Tylospora fibrillosa (Burt) Donk: moss-covered, decorticated Pic, decay 2, diam. $29 \mathrm{~cm}(H K$ 26075).

Tyromyces canadensis Overh. ex J. Lowe (Antrodiella canadensis (Overh.) Niemelä) (HK 26066): decorticated L driftwood, decay 1, diam. $21 \mathrm{~cm}$. Hyphal system apparently monomitic, context with thick-walled (walls up to $1 \mu \mathrm{m}$ ), 3.5-4.5(-5) $\mu \mathrm{m}$ wide clamped hyphae intermixed with thinner-walled hyphae with refractive finger-like appendices (like in $T$. chioneus), tramal hyphae, thin-walled, $2.5-3 \mu \mathrm{m}$ in diam., parallel, glued together, basidia short, 9-12 X 4-5 $\mu \mathrm{m}$ with four, up to $3 \mu \mathrm{m}$ long sterigmata, cystidioles fusoid, $8-9 \times 3.5-5 \mu \mathrm{m}$, spores ellipsoid to subglobose, thin-walled, CB-, inamyloid, 2.4-3.1 × (1.7-)2-2.6(-2.8) $\mu \mathrm{m}, \mathrm{L}_{\mathrm{m}}=2.8 \mu \mathrm{m}$, $\mathrm{W}_{\mathrm{m}}=2.3 \mu \mathrm{m}, \mathrm{Q}=1.1-1.5, \mathrm{Q}_{\mathrm{m}}=1.2(\mathrm{n}=30)$

The spores are small if compared with the sizes given by Niemelä (2005), but fit well with those given by Ryvarden and Melo (2014). PFI.

Tyromyces chioneus (Fr.) P. Karst.: decorticated Al, decay 3, diam. $4 \mathrm{~cm}$ (HK 26044), B branch (SVER 58486).

Vararia investiens (Schwein.) P. Karst.: corticated L branch, decay 1, diam. $2.5 \mathrm{~cm}$ (HK 26093).

Veluticeps abietina (Pers. Fr.) Hjortstam \& Telleria (Colymnocystis abietina (Pers. : Fr.) Pouzar): decorticated Pic, decay 1, diam. $9.5 \mathrm{~cm}(H K$ 26249).

Xylodon brevisetus (P. Karst.) Hjortstam \& Ryvarden (Hyphodontia breviseta (P. Karst.) J. Erikss.): Pic trunk (SVER 58801).

Xylodon crustosus (Pers.) Chevall. (Hyphodontia crustosa (Pers. : Fr.) J. Erikss.): decorticated B, decay 3, diam. $4 \mathrm{~cm}$ (HK 26135).

Xylodon rimosissimus (Peck) Hjortstam \& Ryvarden (Hyphodontia rimosissima (Peck) Gilbn.): Psy branch (SVER 58706).

Xylodon spathulatus (Schrad.) Kunze (Hyphodontia spathulata (Schrad.) Parmasto): Psy branch (SVER 58897).
Xylodon aff. spathulatus (Schrad.) Kuntze (Hyphodontia aff. spathulata (Schrad.) Parmasto) (HK 26155).: decorticated Al, decay 3, diam. $3.5 \mathrm{~cm}$. Basidiocarp resupinate, relatively thick, cream coloured, odontioid with cylindrical, basally normally flattened, apically often furcate, up to $1 \mathrm{~mm}$ long aculei, margin distinct; hyphal system monomitic, tramal hyphae clamped, with thickened walls, 2-3 $\mu \mathrm{m}$ wide, covered with small crystals, cystidia or gloeocystidia none, but spear-shaped, apically roundish, very thinwalled cystidioles fairly common, especially in apical apices of the aculei, 35-41 $\times 4-5 \mu \mathrm{m}$, basidia cylindrical, 24-30 $\times 4-4.5 \mu \mathrm{m}$, with four, up to $4 \mu \mathrm{m}$ long sterigmata, spores broadly ellipsoid, 4.6-5.2 × 3.7-4.2 $\mu \mathrm{m}, \mathrm{L}_{\mathrm{m}}=4.9 \mu \mathrm{m}, \mathrm{W}_{\mathrm{m}}=$ $3.9 \mu \mathrm{m}, \mathrm{Q}=1.1-1.4, \mathrm{Q}_{\mathrm{m}}=1.3(\mathrm{n}=30)$.

The spores fit well with Xylodon spathulatus, but our specimen has neither gloeocystidia nor capitate hyphal ends, but spear-shaped hyphal ends.

\section{List 2 (cantharelloid, clavarioid and hericioid fungi)}

Artomyces pyxidatus (Pers.) Jülich: Psy (AGS 12613).

Auriscalpium vulgare Gray: Psy cone (SVER 58536).

Cantharellus cibarius Fr.: soil in mixed forest (SVER 58628).

Clavaria argillacea Pers.: soil in meadow ( $A G S$ 12667).

Clavaria falcata Pers.: soil in river valley (AGS 12717).

Clavaria fragilis Holmsk.: soil in meadow (AGS 12684).

Clavaria fumosa Pers.: soil and mosses in spruce forest (AGS 12741).

Clavaria sphagnicola Boud.: mosses in river valley (AGS 12762)

Clavariadelphus aff. borealis V.L. Wells \& Kempton: mosses in mixed forest (AGS 12698). 
Clavariadelphus ligula (Schaeff.) Donk: fallen Psy needles (AGS 12624).

Clavariadelphus pistillaris (L.) Donk: soil, under spruces (AGS 12690).

Clavariadelphus sachalinensis (S. Imai) Corner: fallen needles under L and Psi (AGS 12722).

Clavicorona taxophila (Thom) Doty: litter in river valley ( $A G S$ 12776).

Clavulina cinerea (Bull.) J. Schröt.: soil in meadow and river valley ( $A G S$ 12650).

Clavulina coralloides (L.) J. Schröt.: soil in meadow, under grasses (AGS 12683).

Clavulinopsis corniculata (Schaeff.) Corner: soil under grasses ( $A G S$ 12602).

Clavulinopsis helvola (Pers.) Corner: mosses in meadow (AGS 12705).

Clavulinopsis luteoalba (Rea) Corner: mosses and fallen Psy needles (AGS 12658).

Hericium coralloides (Scop. : Fr.) Pers.: corticated B (HK 26194), B (SVER 58770).

Hydnellum aurantiacum (Batsch) P. Karst.: soil in Psy dominated forest (SVER 59119).

Hydnellum ferrugineum (Fr.) P. Karst.: soil in mixed forest (SVER 59145).

Hydnellum peckii Banker: soil in Pic forest in river valley (SVER 59184).

Hydnum repandum L.: soil in Pic forest (SVER 59220).

Hydnum cf. umbilicatum Peck: soil and mosses in mixed forest (SVER 59199).

Lentaria byssiseda Corner: Pic braches on ground (AGS 12879).

Lentaria dendroidea (O.R. Fr.) J.H. Petersen: mosses (AGS 12674).
Lentaria cf. subcaulescens (Rebent.) Rauschert: moss-covered Pic trunk (AGS 12608).

Macrotyphula fistulosa (Holmsk.) R.H. Petersen: buried $\mathrm{B}$ twigs in litter and inside mosses $(A G S$ 12709).

Macrotyphula juncea (Alb. \& Schwein.) Berthier: decaying leaves and litter ( $A G S$ 12753).

Mucronella bresadolae (Quél.) Corner: B, inside the bark tube, decayed by Inonotus obliquus (AGS 12643).

Mucronella calva (Alb. \& Schwein.) Fr. (incl. Mucronella flava Corner): Psy, fallen trunk ( $A G S$ 12730).

Multiclavula corynoides (Peck) R.H. Petersen: soil covered by algae (AGS 12851).

Multiclavula vernalis (Schwein.) R.H. Petersen: soil covered by mosses (AGS 12790).

Pterula gracilis (Desm. \& Berk.) Corner: decaying grasses and fallen leaves (AGS 12634).

Ramaria abietina (Pers.) Quél.: needle litter under Pic, Psy, L (AGS 12784).

Ramaria corrugata (P. Karst.) Schild: needle litter under Pic (AGS 12857).

Ramaria eumorpha (P. Karst.) Corner: needle litter and mosses in mixed forest (AGS 12695).

Ramaria flaccida (Fr.) Bourdot: needle litter and mosses in mixed forest ( $A G S$ 12746).

Ramaria cf. flavescens (Schaeff.) R.H. Petersen: soil in mixed forest (AGS 12622).

Ramaria flavobrunnescens (G.F. Atk.) Corner: soil in mixed forest (AGS 12712).

Ramaria gracilis (Pers.) Quél.: needle litter in Psy forest (AGS 12805).

Ramaria suecica (Fr.) Donk: needle litter in Pic forest (AGS 12828). 
Ramariopsis biformis (G.F. Atk.) R.H. Petersen: soil under grasses in meadow and deciduous forest (AGS 12765).

Ramariopsis subarctica Pilát: mosses on bog border (AGS 12687).

Sarcodon imbricatus (L.) P. Karst.: soil in mixed forest (SVER 59031).

Sarcodon scabrosus (Fr.) P. Karst.: soil in mixed forest ( SVER 58906).

Typhula capitata (Pat.) Berthier: Calamagrostis, dead stems ( $A G S$ 12758).

Typhula caricina P. Karst.: Carex, dead leaves (AGS 12679)

Typhula chamaemori L. Holm \& K. Holm: Rubus chamaemorus, dead leaves (AGS 12646).

Typhula crassipes Fuckel: dead herbs and leaves (AGS 12813).

Typhula culmigena (Mont. \& Fr.) Berthier: dead herbs (AGS 12847).

Typhula erythropus (Pers. : Fr.) Fr.: B, Pop, dead leaves ( $A G S$ 12708).

Typhula graminum P. Karst.: grasses, dead leaves (AGS 12772).

Typhula hyalina (Quél.) Berthier: dead grasses, herbs and tree leaves ( $A G S$ 12636).

Typhula incarnata Lasch ex Fr.: grasses ( $A G S$ 12808).

Typhula lutescens Boud.: dead herbs (AGS 12891).

Typhula micans (Pers.) Berthier: dead herbs (AGS 12743).

Typhula phacorrhiza (Reichard : Fr.) Fr.: dead tree leaves and herbs (AGS 12616).

Typhula sclerotioides (Pers.) Fr.: dead herbs, like Aconitum (AGS 12736).
Typhula setipes (Grev.) Berthier: B, S, fallen leaves (AGS 12654).

Typhula subvariabilis Berthier: Sor, dead leaves (AGS 12871).

Typhula uncialis (Grev.) Berthier: Epilobium, dead stems ( $A G S$ 12827).

Typhula variabilis Riess: Urtica, dead stems (AGS 12750).

\section{Discussion}

Altogether 248 taxa were collected. The most species rich morph group was the corticioid fungi with 120 taxa, followed by polypores with 65 taxa. The ratio polypores/corticioids (including the genera Boletopsis, Thelephora, Tomentella, Tomentellopsis) is 0.54 and without the Thelephoraceae 0.58 .

The morph groups cantharelloid, clavarioid and hericioid fungi (List 2) include 63 species. The most species rich genus is Typhula with 17 species. The most species rich genera of corticioids and polypores are Hyphodontia sensu lato (10 species), Tubulicrinis (9), Phellinus (8), Phlebia (8) and Peniophora (6).

Most commonly noted ten species (Fomes fomentarius 51 notes, Peniophora aurantiaca 26, Fomitopsis pinicola 22, F. rosea 12, Amphinema byssoides 11, Daedaleopsis tricolor 10, Inonotus radiatus 10, Ganoderma applanatum 9, Trichaptum abietinum 9 and Hyphoderma setigerum 8) cover $30.6 \%$ of all observations.

There are some surprising results in our study. For instance, Resinicium bicolor (Alb. \& Schwein.) Parmasto, is normally very common in boreal forests, but was absent here. Also the species of the genera Botryobasidium, Sistotrema and Trechispora were surprisingly rare. We do not have any explanation for it, since there were enough suitable habitats and substrates. Perhaps only the weather conditions were not suitable in these years to support the fruit body formation. Many species also had unusual small spores, even if they otherwise were "normal" looking.

The area comprises several old-growth forest species which are absent or at least very rare in managed forest. Some of these species, like Fomitopsis rosea, can grow also on constructed 
wood, like collapsed hay barns. However, the frequency in old-growth/primeval forests is much higher than elsewhere. We collected also outside the nature reserve, where the forests were even older than in the nature reserve. Therefore it would be very important to protect the forests along the Podkammennaja Tunguska River also outside the borders of the nature reserve.

Acknowledgements: We thank J. Päivärinta and P. Rusanen (Helsinki) for pleasant companionship and help in collection. We also thank S. I. Polunina and A. N. Shuman (Krasnoyarsk) for the specimens they collected in 2014. I. V. Stavishenko (Ekaterinburg) identified a part of the collections - we are very grateful for her help. The climate data was compiled by Stefan Fronzek (Helsinki) and the director of the Central Siberian Nature Reserve, P. V. Kochkarev, gave very valuable help in organization the expedition. Both are warmly acknowledged.

\section{References}

Ahti, T., Hämet-Ahti, L. \& Jalas, J. 1968: Vegetation zones and their sections in northwestern Europe. Ann. Bot. Fennici 5: 169-211.

Bernicchia, A. \& Górjon, S.P. 2010: Corticiaceae s.1. Fungi Europaei 12: 1-1008.

Czerepanov, S.K. 1995: Vascular plants of Russia and adjacent states (the former USSR). - Cambridge University Press. $516 \mathrm{pp}$.

Eriksson., J. Hjortstam, K. \& Ryvarden, L. 1981: The Corticiaceae of North Europe 6. Phlebia to Sarcodontia. - Fungiflora. Oslo. Pp. 1051-1276.

Eriksson, J., Hjortstam, K. \& Ryvarden, L. 1984: The Corticiaceae of North Europe 7. Schizopora to Suillosporium - Fungiflora. Oslo. Pp. 1281-1449.

Eriksson, J. \& Ryvarden, L. 1973: The Corticiaceae of North Europe 2. Aleurodiscus to Confertobasidium. Fungiflora. Oslo. Pp. 60-286.

Eriksson, J \& Ryvarden, L. 1975: The Corticiaceae of North Europe 3. Coronicium to Hyphoderma. Fungiflora. Oslo. Pp. 287-546.

Ghobad-Nejhad, M., \& Hallenberg, N. 2012: Multiple evidence for recognition of Phlebia tuberculata, a more widespread segregate of Phlebia livida (Polyporales, Basidiomycota) - Mycol. Progress 11: 27-35.

Hallenberg, N. 1985: The Lachnocladiaceae and Coniophoraceae of North Europe. - Fungiflora. Oslo. 96 pp.

Harris, I., Jones, P.D., Osborn, T.J. \& Lister, D.H. 2014: Updated high-resolution grids of monthly climatic observations the CRU TS3.10 Dataset. - Int. J. Climatol. 34: 623 - 642. doi: 10.1002/joc.3711.

Hjortstam, K. \& Ryvarden, L. 2009: A checklist of names in Hyphodontia sensu stricto - sensu lato and Schizopora with new combinations in Lagarobasidium, Lyomyces, Kneiffiella, Schizopora, and Xylodon. - Synopsis Fungorum 26: 33-55.

Kotiranta, H. 1995: A preliminary mycobiota (Aphyllophorales, Fungi) of timberline forests in the West Siberian plateau. - Arctic Centre Publications 7: 78-95.
Kotiranta, H., Larsson, K.H. \& Saarenoksa, R. \& Kulju, M. 2011: Tretomyces gen. novum, Byssocorticium caeruleum sp. nova, and new combinations in Dendrothele and Pseudomerulius (Basidiomycota). - Ann. Bot. Fennici 48: 37-48.

Kotiranta, H. \& Mukhin, V.A. 1998: Polyporaceae and Corticiaceae of an isolated forest of Abies nephrolepis in Kamchatka, Russian Far East. - Karstenia 38: 6980 .

Kotiranta, H. \& Mukhin, V.A. 2000: Aphyllophorales (Basidiomycetes) of Tiksi, Republik of Sakha (Yakutia), Northeast Siberia. - Karstenia 40: 65-69.

Kotiranta, H., Mukhin, V.A., Ushakova, N. \& Dai, Y-C. 2005: Polypore (Aphyllophorales, Basidiomycetes) studies in Russia 1. South Ural. - Ann. Bot. Fennici 42: 427-451.

Kotiranta, H. \& Niemelä, T. 1996: Uhanalaiset käävät Suomessa. Toinen, uudistettu painos. Threatened polypores in Finland. Second revised edition. (in Finnish, with English summary). - Ympäristöopas 10: $1-184$.

Kotiranta, H. \& Saarenoksa, R. 1990: Reports of Finnish corticolous Aphyllophorales (Basidiomycetes). Karstenia 30: 43-69.

Kotiranta, H. \& Saarenoksa, R. 2005: The genus Athelopsis (Aphyllophorales, Corticiaceae) in Finland. - Ann. Bot. Fennici 42: 335-342.

Kotiranta, H., Saarenoksa, R. \& Kytövuori, I. 2009: Aphyllophoroid fungi of Finland. A check-list with ecology, distribution, and threat categories. - Norrlinia 19: 1-223.

Kotiranta, H., Ushakova, N. \& Mukhin, V.A. 2007: Polypore (Aphyllophorales, Basidiomycetes) studies in Russia. 2. Central Ural. - Ann. Bot. Fennici 44: 103-127.

Mukhin, V.A. 1993: Biota ksilotrofnykh Bazidiommitsetov zapadno-sibirskoi ravniny - Rossiskaya Akademiya Nauk. Ekaterinburg. 231 pp.

Mukhin, V.A. \& Kotiranta, H. 2001: Wood-decaying fungi of northernmost forests in river Khatanga basin. - Mikologia i Fitopatologia 35: 41-47.

Niemelä, T. 2005: Käävät, puiden sienet. Polypores, lignicolous fungi (in Finnish, with English summary). - Norrlinia 13: 1-320.

Parmasto, E. 1963: K flore gribov polyostrova Kamchatki - Issledovanie Prirody Dal'nego Vostoka 1: 221-289.

Parmasto, E. 1965: Corticiaceae U.R.S.S. I. Descriptiones taxum novarum Combinationes Novae. - Eesti NSV Teaduste Akadeemia Toimetised 14: 220-233.

Ryvarden, L. \& Gilbertson, R.L. 1994: European Polypores 2. - Synopsis Fungorum 7: 388-743.

Ryvarden, L. \& Melo, I. 2014: Poroid fungi of Europe. Synopsis Fungorum 31: 1-455.

Shiryaev, A.G., Kotiranta, H., Mukhin, V.A., Stavishenko, I.V. \& Ushakova, N.V. 2010: Aphyllophoroid fungi of Sverdlovsk Region, Russia. Biodiversity, distribution, ecology and the IUCN threat categories. Goshchitskiy Publisher. Ekaterinburg. 304 pp.

Stavishenko, I.V. 2011: Aphyllophoraceous fungi of the Nature Reserve Malaya Sosva (Western Siberia). Mikologia i Fitopatologia 45: 142-157.

Yurchenko, E.O. 2010: The genus Peniophora (Basidiomycota) of Eastern Europe. - Belorusskaya nauka. Minsk. 338 pp. 